**THIS PRE-PRINT IS A WORKING PAPER THAT HAS NOT BEEN PEER-REVIEWED**

\title{
Fatalism in the Fight against COVID-19: Implications for Mitigation and Mental Health
}

\author{
Joseph Hayes and Laura Clerk \\ Department of Psychology, Acadia University
}

\begin{abstract}
Author Note
This study was not preregistered. De-identified data and analysis script are posted at https://osf.io/sx7g2/; access to the data is limited to qualified researchers. The materials used in the study, as well as supplemental data analyses are also publicly available at the same link. The study received approval by the Research Ethics Board of Acadia University (REB 19-29). We have no known conflict of interest to disclose. Financial support for this project was provided by a Harrison McCain Emerging Scholar Grant awarded to the first author.

Correspondence concerning this article should be addressed to Joseph Hayes, Acadia University, Department of Psychology, 18 University Ave, Wolfville, NS, B4P 2R6, Canada. Email: joseph.hayes@acadiau.ca.
\end{abstract}




\begin{abstract}
This research assessed fatalism toward COVID-19 and its role in behavioral intentions to support mitigation efforts (e.g., social distancing) and mental well-being. A COVID-19 fatalism measure was developed, and a messaging manipulation (fatalistic vs. optimistic vs. no message) was created to causally affect fatalism scores. Support for mitigation efforts and negative affect (anxiety, fear, depression, and insecurity) were measured to examine the consequences of fatalism toward COVID-19. Results showed that the fatalistic messaging condition increased fatalism whereas the optimistic message reduced it. The effects of the messaging manipulation were also apparent in the downstream measures of support for mitigation and negative affect through the mediator of fatalism toward COVID-19. Specifically, fatalism negatively predicted intentions to support mitigation. Regarding mental health, fatalism was positively associated with depression but negatively associated with fear and insecurity. Implications for COVID-19 mitigation efforts and mental health in the face of the coronavirus pandemic are discussed. Keywords: COVID-19, Fatalism, Social Distancing, Mental Health
\end{abstract}




\section{Statement of Relevance}

This research examined two critically important issues arising from the novel coronavirus pandemic: adherence to mitigation efforts (e.g., social distancing) and mental health. The key psychological factor assessed in relation to each of these issues is fatalism toward the pandemic. Fatalism is a response to overwhelming threats that appear uncontrollable. The response entails withdrawing efforts to control the threat due to feelings of powerlessness. This withdrawal may function to reduce fear and anxiety stemming from the uncontrollable threat, but it increases depression. Our research examined how fatalism toward COVID-19 affected self-reported negative emotions and behavioral intentions to support mitigation efforts. A messaging manipulation aimed at either increasing or decreasing fatalism toward COVID-19 was found to influence support for mitigation and emotional well-being, but only indirectly by influencing fatalism. These results have important implications for ongoing mitigation efforts and for understanding the mental health fallout of the pandemic. 


\section{Fatalism in the Fight against COVID-19: Implications for Mitigation and Mental Health}

Since March $11^{\text {th }}, 2020$, the world has been gripped by a deadly pandemic (World Health Organization, 2020). At the time of writing, over three million people have been infected worldwide, and more than 200 thousand people have died (Worldometer, 2020; April 27 ${ }^{\text {th }}$ ). Mitigation efforts aimed at reducing the spread of the virus have been in place for more than a month. Schools and businesses are closed, and people are asked to practice social distancing by staying at home, avoiding crowds, and maintaining a minimum distance between themselves and others while in public (Centres for Disease Control and Prevention, 2020). Yet the global spread of COVID-19 shows little sign of abatement.

As if the death-toll of the pandemic is not enough, the mental toll of the pandemicincluding that associated with mitigation efforts - cannot be ignored. Millions of people have lost their job and are now facing unprecedented financial strain (Mutikani, 2020; Department of Labor, 2020). People are isolating at home, often in closed quarters, and are lacking many of the things that usually offer meaning and purpose to life (e.g., social contacts, freedom of movement, sports and entertainment). Domestic violence is on the rise (Taub, 2020). Parents are struggling to manage childcare and homeschooling while also trying to work from home (Cooney, 2020). The list goes on. To make matters worse, it appears that this may be the new normal until a vaccine is developed, which will likely take a year or more (Boyle, 2020).

Under such dire circumstances, it can be difficult to carry on. For many, the strain of the new normal appears impossible to manage. Rather than continue to struggle with mitigation efforts, many people may be willing to give up and submit to the deadly pandemic or risk their health and safety by attempting to return to the "old" normal. Given that most cases appear to be relatively mild, the appeal of letting go, and allowing the virus to wash through the population 
may seem more attractive than maintaining efforts to mitigate its spread, especially where the effects of mitigation efforts are slow to materialize. The purpose of the current research was to assess fatalism toward COVID-19 at a pivotal point in America's efforts to fight the virus. Moreover, aimed to examine the effect of different media messages on feelings of fatalism, and sought to gauge the consequences of COVID-19 fatalism on support for continued mitigation efforts and mental well-being.

\section{Fatalism toward COVID-19}

Fatalism is the belief that one's actions have little or no significant impact on important outcomes (Zimbardo \& Boyd, 1999). People who are high in fatalism tend not to engage futureoriented planning, expend little effort in trying to achieve desirable goals, and are generally resigned to fate. In other words, they are willing to let external forces take over.

Mental Health. Fatalism is strongly associated with depression and hopelessness (Seligman, 1975; Zimbardo \& Boyd, 1999). However, it can also function to reduce fear and anxiety stemming from insurmountable threats (Hayes et al., 2016). Struggling to control outcomes that appear intractable triggers anxious motivational conflict (Carver \& Scheier, 1998; Gray \& McNaughton, 2000), while choosing to let go of them can reduce this anxiety by eliminating the tension produced by wanting to control something uncontrollable (Hayes et al., 2017). Fatalism in the fight against COVID-19 may be especially likely given that mitigation efforts are difficult, costly, have no clear end-date, and do not show clearly discernible causal effects. Thus, becoming fatalistic in the fight against COVID-19 can be an attractive way of reducing concerns about the pandemic. Importantly, however, this method of palliation has broader negative consequences for mental health by promoting depression and generalized disengagement from life (Hayes et al., 2016). 
Commitment to Mitigation Efforts. Another trouble with fatalism is that it reduces motivation and planful self-regulation (Hayes et al., 2016), which may be especially problematic vis-à-vis COVID-19 because it may undermine the principal means of addressing the pandemic — namely, social distancing. Becoming fatalistic about COVID-19 may lead people to ignore public health recommendations (e.g., "we're all going to get this virus anyway, so why stay at home and suffer?"), which is dangerous for the general public as well as the fatalistic individual as it increases the risk that they will become infected and spread the virus by not taking proper precautions. Understanding factors that contribute to COVID-19 fatalism, and how we can reduce these factors, is therefore imperative if collective mitigation efforts are to be successful.

Media Messaging. One factor that may be particularly important in creating fatalism toward COVID-19 is the way in which it is presented in the media. Indeed, nearly every news story currently being disseminated to the public appears related to the pandemic in some manner or another, and the information is often dire or shocking. While many of these messages seek to affirm the importance of collective action and the ironic sense of community that can come from social distancing for the well-being of others (e.g., \#AloneTogether; Harrop, 2020), other messages often directly promote fatalism by claiming that the spread of the disease is inevitable (Slaughter, 2020). These messages often voice concern about the long-term economic impact of staying at home and shuttering businesses, suggesting that the cost associated with continued mitigation efforts is far greater than the cost of the virus (Hilton, 2020; Singer \& Plant, 2020). U.S. President Donald Trump appears to share these concerns, tweeting on March $23^{\text {rd }}$ "WE CANNOT LET THE CURE BE WORSE THAN THE PROBLEM ITSELF” (Trump, 2020). Although these messages are very clearly anti-mitigation, we suspect they may be most effective 
in reducing support for mitigation efforts when they instill a sense of fatalism toward COVID19. Indeed, fatalism and inaction go hand-in-hand.

\section{Study Overview}

The purpose of the current research was to assess levels of fatalism toward COVID-19, to understand what factors influence this construct, and to examine the consequences of fatalism for mental health and support for COVID-19 mitigation efforts. Accordingly, we developed a selfreport measure of fatalism toward COVID-19 and collected an online survey. To assess how COVID-19 fatalism can be causally influenced, we designed a fatalistic message arguing that the pandemic is unstoppable and that mitigation efforts may do more harm than good (cf., Hilton, 2020; Singer \& Plant, 2020; Slaughter, 2020; Trump, 2020). The fatalistic message intended to mimic those presented in the media or shared by prominent figures that the general public has been exposed to throughout the pandemic. For comparison, we created an optimistic message that emphasized the effectiveness of mitigation and the connectedness that can come from tackling the pandemic collectively. We expected the fatalistic message to increase fatalism and the optimistic message to reduce it.

To assess the consequences of COVID-19 fatalism, at the end of the study we assessed support for mitigation and negative emotionality. Regarding support for mitigation efforts, we expected fatalism to be associated with reduced support for these efforts. Moreover, we hypothesized that our fatalistic and optimistic messaging conditions would influence support for mitigation by virtue of affecting fatalism (i.e., mediation). Regarding negative emotion, we expected fatalism to be positively associated with depression (Zimbardo \& Boyd, 1999), but negatively associated with anxiety given evidence that fatalism in the face of insurmountable threats can reduce anxiety (Hayes et al., 2016). 


\section{Method}

\section{Participants and Design}

In keeping with open science practices, we report all measures and manipulations included in the study. Full study materials are available online at https://osf.io/sx $7 \mathrm{~g} 2 /$. We also explain how sample size was determined and report all data exclusions.

To determine minimum sample size requirements to confidently test our hypotheses, we conducted an a priori power analysis using $\mathrm{G}^{*}$ Power (Faul et al., 2007), and sought at least $80 \%$ power (with an alpha of .05) to detect a small effect (i.e., $f=.10$ to $.24 ; d=.20$ to .49 ). The number of participants required to detect the lowest end of this range $(f=.10 ; d=.20)$ using these criteria in a one-way ANOVA with three conditions yielded the highest estimate $(N=969)$, so we strove to obtain a sample size that approximated this number.

Participants were 1025 people recruited online through Amazon’s Mechanical Turk. They were randomly assigned to one of three conditions in a between-subjects design. The only requirement for participation was United States residence. Exclusions included 149 participants who failed an attention check item asking them to leave a question blank (i.e., Please do not answer this question, it is here to see if you are paying attention.), 19 who did not correctly answer at least two (of four) multiple-choice questions about the contents of the article that they read, and six others for failing to complete all dependent variables for our main analyses. The total number of participants after exclusions was 851 (fatalistic $n=274$, optimistic $n=291$, no message $n=286$ ), which fell short of our sample size goal. Nevertheless, a post-hoc sensitivity analysis revealed that we retained $80 \%$ power to detect small effects $(f=.11, d=.21$; and $95 \%$ power to $\operatorname{detect} f=.13, d=.27$ ), so we were confident in proceeding with our analyses without collecting more data. 
Participants ranged in age from 18 to $78\left(M_{\text {age }}=41.0, S D_{\text {age }}=14.0\right)$, and gender balance was roughly equal $($ female $=443$, male $=391$, other $=5$, prefer not to disclose $=6$ ).

\section{Measures and Procedure}

The study was conducted on March $27^{\text {th }}, 2020$, eleven days into the initial mitigation period aimed at providing 15-days to slow the spread of the novel coronavirus. The study was reviewed by an institutional research ethics board and was deemed to pose no more than minimal risk, as the messages used in the manipulation were comparable to those appearing in the popular media and public discourse. Participants were informed that the study was an investigation of personality, attitudes, and opinions. They were not told that the purpose of the study was related to COVID-19 until the debriefing. Upon consenting to participate, respondents began by complete a series of demographic questions, followed by three brief personality questionnaires.

Demographic and Personality Variables. Demographic items included age, gender, household income, education, and political orientation (among others, see online supplement for complete list of demographic items). We measured three personality factors that seemed like plausible candidates for influencing fatalism. Specifically, we assessed self-esteem (Rosenberg, 1965) and trait sensitivity to rewards and punishments (BAS and BIS; Carver \& White, 1994) given that these variables influence reactions to threat (see Jonas et al., 2014; Pyszczynski et al., 2004). Exploratory analyses controlling for demographic and personality factors and exploratory tests of moderation are presented in the Supplementary Online Material (SOM; see https://osf.io/sx7g2/).

Messaging Condition. Participants were randomly assigned to one of three messaging conditions. In the control condition, participants read no message and simply proceeded to a series of questions related to their attitudes and opinions about the COVID-19 pandemic. By 
contrast, those in the fatalistic and optimistic messaging conditions read a brief opinion piece before proceeding to these questions. The essays began with a threatening paragraph outlining the severity of the COVID-19 pandemic:

COVID-19 is a killer. It has already killed more than 25,000 people worldwide and will likely kill many hundreds of thousands more. Making things worse, it is an invisible killer. We cannot see it, and we cannot even know when we have it on our hands. The only way that we know to keep it at bay is to stay away from each other. Social (or physical) distancing measures have been in place across the Western World for nearly two weeks. But the spread of COVID-19 rages on.

For participants in the fatalistic condition, the essay went on to describe how social distancing can only be a temporary fix, and that the virus will remain problematic until we develop a vaccine, which will not happen for 18-24 months. The article then struck a fatalistic chord by asking whether people are truly willing to engage social distancing for two years. The author indicates that the virus is unstoppable and that he would rather let it run its course so that we can get back to normal sooner than later.

Participants in the optimistic condition read an article that began with the same opening paragraph but then proceeded to argue that social distancing is effective. The author pointed toward China and South Korea as examples of its effectiveness. The message is optimistic but nevertheless realistic, suggesting that social distancing will not eradicate the virus but will buy time so that a vaccine can be developed within 18-24 months. The author concludes by indicating that he is willing to do his part to prevent the spread of the virus. Finally, he appeals to the togetherness that collective social distancing can offer (see SOM for the full text of both messaging conditions). 
Participants in the fatalistic and optimistic conditions then proceeded to complete five simple reading comprehension questions that were included to ensure adequate processing of the message. Four of these questions were multiple-choice, whereas one was an open-ended item asking participants to indicate the overall theme of the article. Only participants who correctly answered at least two of the four multiple choice questions were retained for data analyses.

Specific Worries. Next, participants completed a 7-item scale assessing their specific worries related to the COVID-19 crisis. These items were included for exploratory purposes. They assessed worries about death and finances for the self, close others, and strangers. The final item assessed concern for the economy (see SOM for exploratory analyses of these items).

Fatalism toward COVID-19. Participants then completed a 16-item scale assessing fatalism toward COVID-19 ( $\alpha=.94$; see Table 1 for complete item-details). This was our main dependent variable, and consisted of seven positively keyed items ("Since whatever will be will be, it doesn't really matter what I do to try to stop COVID-19") and nine negatively-keyed items (indicative of self-efficacy; e.g., "It is within my power to help reduce the spread of COVID19"). Participants rated their agreement with each item using a 7-point Likert scale $(1=$ strongly disagree; 7 = strongly agree).

Behavioral Intention to Support Mitigation Efforts. Immediately after the fatalism questionnaire, we included an 11-item scale assessing behavioral intentions to support mitigation efforts ( $\alpha=.86$, see SOM for complete item-details). Participants again responded using the 7point scale. The items were geared predominantly toward intentions to engage social distancing (e.g., I plan to keep my distance from others) and to remain isolated (e.g., I plan to stay isolated for as long as it is required), but also assessed support for mitigation efforts more broadly (e.g., I support lockdown efforts aimed at reducing the spread of COVID-19). 
Emotional Distress. Finally, after rating their support for mitigation efforts, participants completed a brief emotion measure to gauge their emotional well-being. The measure consisted of 20 items assessing four different emotions (5-items each): anxiety $(\alpha=.84)$, fear $(\alpha=.95)$, depression $(\alpha=.93)$, and insecurity ( $\alpha=.87$; see SOM for complete item-details). Although our hypotheses were specifically related to anxiety and depression, we included items assessing fear and insecurity for exploratory purposes. Participants rated the extent to which they were currently experiencing these emotions using a 7 -point scale $(1=$ not at all; $7=$ very much $)$. Upon completion, participants were thanked for their participation and fully debriefed.

\section{Results}

Deidentified data and analysis script for all analyses reported below are available online at https://osf.io/sx7g2/. A document containing supplemental exploratory analyses that accompany the main findings can be found by following the same link.

\section{Fatalism toward COVID-19}

To test our hypothesis about the effect of messaging condition on fatalism toward COVID-19, we conducted a one-way ANOVA on the fatalism scores. Results showed a significant effect of condition, $F(2,848)=11.16, p<.001, \eta^{2}=.03$ (see Figure 1). Consistent with hypotheses, pairwise comparisons revealed that the fatalistic message increased fatalism relative to the no message control condition, $t(848)=2.04, p=.041, d=.17$, whereas the optimistic message reduced fatalism relative to no message, $t(848)=-2.68, p=.007, d=.22$.

\section{Behavioral Intentions to Support Mitigation Efforts}

Our next analysis examined the consequences of COVID-19 fatalism for behavioral intentions to support mitigation efforts. First, a bivariate correlation between these variables showed a highly significant association, $r(851)=-.78, p<.001$. Thus, higher levels of fatalism 
toward COVID-19 were associated with lower behavioral intentions to support mitigation efforts. We then examined the effect of our messaging manipulation on support for mitigation efforts. A one-way ANOVA on support for mitigation with message condition as the independent variable revealed a significant effect, $F(2,848)=4.33, p=.013, \eta^{2}=.01$ (see Figure 2). Pairwise comparisons revealed that whereas the optimistic message increased support for mitigation efforts relative to no message, $t(848)=2.57, p=.010, d=.21$, the fatalistic message had no effect on support for mitigation, $t(848)=-.00, p=.997, d=-.00$. However, our main hypothesis regarding the effect of messaging condition on support for mitigation was that it would be mediated by fatalism toward COVID-19. Thus, we tested the indirect effect of our messaging manipulation on support for mitigation efforts through the hypothesized mediator of fatalism.

Accordingly, we used Hayes' (2018) PROCESS macro to regress support for mitigation on messaging condition (dummy-coded to compare the fatalistic message with control in code 1 and the optimistic message with control in code 2) through the mediator of COVID-19 fatalism (Model 4, 5000 bootstrap resamples). This analysis showed that the fatalistic message reduced support for mitigation indirectly by increasing fatalism toward COVID-19, $b=-.13,95 \%$ confidence interval (CI) [-.266, .003], whereas the optimistic message increased support for mitigation by reducing fatalism, $b=.17,95 \%$ CI $[.051, .299]$. After accounting for these indirect effects, messaging condition still exerted a significant direct effect on support for mitigation efforts, $F(2,847)=3.31, p=.037, \eta_{p}{ }^{2}=.003$. Interestingly, pairwise comparisons showed that whereas the direct effect of the optimistic message was not significant, $t(847)=.77, p=.443, d=$ .04 , the fatalistic message now revealed a significant direct effect, $t(847)=.2 .52, p=.012, d=$ 
.13, such that participants increased their support for mitigation efforts after reading the fatalistic message relative to no message (see Figure 3 for a full path model).

\section{Emotional Distress}

Finally, we examined the emotional consequences of fatalism toward COVID-19.

Bivariate correlations between fatalism and each of the negative emotions measured in the study are presented in Table 2. Given research showing divergent unique associations between fatalism and anxiety and depression when these highly correlated negative emotions are covaried for each other in statistical analyses (see Hayes et al., 2016; Hayes \& Hubley, 2017), we also examined partial correlations between fatalism and each negative emotion controlling for the others in Table 2. In summary, although the overall correlations show only a significant positive association between fatalism and depression, the partial correlations also show significant negative associations between fatalism and fear and insecurity.

To examine the effect of messaging condition on negative emotionality, we first conducted four separate ANCOVAs on each emotion while controlling for the other three (removing the covariates did not affect these analyses, but see SOM for results without the covariates). These analyses revealed a significant effect on insecurity, $F(2,845)=3.21, p=.041$, $\eta_{p}{ }^{2}=.01$, such that the optimistic message increased insecurity relative to no message, $t(845)=$ $2.52, p=.012, d=.21$, but the fatalistic message did not, $t(845)=1.43, p=.155, d=.12$. There were no overall effects of condition for anxiety, $F(2,845)=1.33, p=.266, \eta_{p}{ }^{2}=.00$, fear, $F(2$, $845)=1.14, p=.320, \eta_{p}{ }^{2}=.00$, or depression, $F(2,845)=.61, p=.545, \eta_{p}{ }^{2}=.00$ (see Figure 4$)$. Nevertheless, given the significant effect of condition on fatalism and the significant partial correlations between fatalism and three of the four negative emotions, we tested for indirect effects of messaging condition on each emotion through the mediator of fatalism using Hayes' 
(2018) PROCESS macro (Model 4, 5000 bootstrap resamples). A full path model is displayed in

Figure 5, and indirect effects are summarized in Table 3 (see SOM for this analysis without the covariates). Overall, this analysis showed that by increasing fatalism toward COVID-19, the fatalistic message indirectly reduced fear and insecurity, but increased depression. The opposite pattern emerged for the optimistic message. By reducing fatalism toward COVID-19, the optimistic message indirectly increased fear and insecurity, but reduced depression.

\section{Discussion}

The results were generally consistent with our hypotheses and offer insights into the role of fatalism in the fight against COVID-19. First, as anticipated, the fatalistic message increased fatalism towards COVID-19 while the optimistic message reduced it. Results for behavioral intentions to support mitigation efforts were partially consistent with hypotheses. As predicted, the optimistic message increased support for mitigation, and fatalism toward COVID-19 mediated this effect. However, the fatalistic message showed no overall effect on support for mitigation. Nevertheless, consistent with hypotheses, this message reduced support for mitigation indirectly by increasing fatalism toward COVID-19. Interestingly, after accounting for this indirect effect, we found that the fatalistic message increased support for mitigation directly (see Figure 3). This pattern offers explanation for why the fatalistic message had no overall effect on support for mitigation—suggesting that the message produced two opposing effects. Whereas some people became fatalistic and thus less supportive of mitigation efforts, others reacted against the message by increasing their support for mitigation. This pattern may be indicative of reactance (Brehm, 1966), wherein people respond to external pressure by asserting their freedom and control. 
Fatalism toward COVID-19 also showed associations with emotional distress, and messaging condition evinced significant indirect effects on negative emotionality by influencing fatalism. First and foremost, fatalism was positively associated with depression. However, when controlling for the other negative emotions assessed in the study, fatalism was also negatively associated with fear and insecurity. These associations are only partially consistent with expectations. We hypothesized a positive association for depression given the withdrawaloriented nature of fatalistically abandoning efforts to engage personal control (which was supported), but a negative association with anxiety given that fatalism can offer defense against intractable threats (Hayes et al., 2016). Results showed no associations between fatalism and anxiety (with or without the covariates). But the associations of fatalism with fear and insecurity may be consistent with our hypothesis that fatalism is an attempt to cope with the intractable nature of the pandemic. In retrospect, anxiety may be more likely when examining distant or abstract threats, whereas clear and present dangers should be more likely to trigger fear-related emotions (McNaughton \& Corr, 2004; Greenberg et al., 1997). These results would need to be replicated to ensure that they are reliable, but the associations with fear and insecurity appear to be consistent with our expectation that fatalism toward COVID-19 offers a means of reducing concerns about the pandemic.

\section{Implications for COVID-19 Mitigation}

The results of the current study have important implications for ongoing efforts to mitigate the spread of COVID-19 by suggesting a pivotal role for fatalism. People are unlikely to adhere to mitigation protocols unless they believe COVID-19 can be eradicated and that their actions (e.g., social distancing, staying at home) are effective in stopping the virus. Messages that paint a bleak picture of the pandemic, or suggest that it may take years to stop if it can be 
stopped at all, may undermine support for mitigation efforts by promoting fatalism (cf., Briscese et al., 2020).

Our results also suggest that messaging about the virus can be an important means of reducing fatalism and thereby increasing support for mitigation. Consistent with this idea, promitigation messages that are inherently anti-fatalistic by drawing clear connections between individual actions and the spread of the virus have been shown to increase intentions to practice social distancing (Lunn et al., 2020). Moreover, messages that promote a duty to care for others (Everett et al., 2020) have also been found to be effective in promoting adherence to mitigation protocols, and these too may function in part by reducing fatalism (see SOM for associations between concern for others, fatalism, and support for mitigation).

\section{Implications for Mental Health}

The current research also has implications for understanding the mental health consequences of the pandemic. Primarily, we found that fatalism toward COVID-19 is positively associated with depression, and that media messaging can influence depression by affecting fatalism. In fact, our fatalistic message was inspired by extent media messages, including Trump's tweet about the cure being worse than the disease. At the time of data collection (March

$27^{\text {th }}$ ), the possibility that Trump would relax restrictions and allow the virus to go unmitigated to save the economy appeared real. We reasoned that this anti-mitigation rhetoric may be effective in reducing support for mitigation, but suspected it would also promote depression and despair by causing many people to feel fatalistic about the pandemic. Our results support this reasoning. However, the data also show that optimistic media messaging can reduce feelings of depression by reducing fatalism toward the COVID-19 pandemic. Thus, the mental well-being of people 
who consume media related to the pandemic hinges in part upon the extent to which the message makes them feel fatalistic (vs. powerful and effective) toward the virus.

Although our sample of online participants is not fully representative of the American public and we could not assess clinical levels of depression, the data nevertheless support growing concerns for the psychological toll of the pandemic (Galea et al., 2020; Garfin et al., 2020). Moreover, whereas stress related to the pandemic may increase depression and anxietyrelated mental health issues (e.g., posttraumatic stress; Bo et al., in press), our data add complexity to the mental health picture by showing that fatalism toward the pandemic can reduce feelings of fear and insecurity. After all, if one concludes that nothing can be done to stop the virus, then there is no sense in worrying about it. Indeed, we maintain that this is the inherent appeal of fatalism in response to intractable threats such as the COVID-19 pandemic. However, fatalism should not be viewed as a healthy solution to the problem. Although additional research is needed to fully understand the ramification of COVID-19 fatalism, it is possible that fatalism toward a pandemic that has completely upended one's life can trigger corresponding feelings of fatalism regarding all life-sustaining activities (see Hayes et al., 2016). If so, fatalism may become a key factor in producing post-pandemic depression and suicide. While mitigation efforts are currently being directed toward saving lives from the physical ravages of COVID-19, these efforts may soon need to pivot toward saving lives from the psychological aftershocks of the crisis. 


\section{Author Contributions}

J. Hayes and L. Clerk developed the study concept. J. Hayes designed the study, and L. Clerk provided critical feedback. Data collection, analysis, and interpretation was performed by J.

Hayes. L. Clerk performed an extensive review of the literature. J. Hayes drafted the manuscript, and L. Clerk provided critical revisions. Both authors approved the final version of the manuscript for submission. 


\section{References}

Bo, H., Li, W., Yand, Y., Wang, Y., Zhang, Q., Cheung, T., Wu, X., \& Xiang, Y. (in press). Posttraumatic stress symptoms and attitudes toward crisis mental health services among clinically stable patients with COVID-19 in China. Psychological Medicine. doi:S0033291720000999

Boyle, Patrick. (2020). Here's why we can't rush a COVID-19 vaccine. Association of American Medical Colleges. https://www.aamc.org/news-insights/here-s-why-we-can-t-rush-covid$\underline{19-\text { vaccine }}$

Brehm J. W. (1966). A theory of psychological reactance. Academic Press.

Briscese, G., Lacetera, N., Macis, M., \& Tonin, M. (2020). Compliance with COVID-19 socialdistancing measures in Italy: The role of expectations and duration [Preprint]. National Bureau of Economic Research. http://www.nber.org/papers/w26916

Carver, C. S., \& Scheier, M. F. (1998). On the self-regulation of behaviour. Cambridge University Press. http://www.doi.org/10.1017/CBO9781139174794

Carver, C. S., \& White, T. L. (1994). Behavioural inhibition, behavioural activation, and affective responses to impending reward and punishment: The BIS/BAS scales. Journal of Personality and Social Psychology, 67(2), 319-333. http://www.doi.org/10.13072/midss.536

Centers for disease control and prevention. (2020). Social distancing, quarantine, and isolation. https://www.cdc.gov/coronavirus/2019-ncov/prevent-getting-sick/social-distancing.html

Cooney, C. I. (2020, April 5). The parents are not all right. Medium. https://gen.medium.com/parents-are-not-ok-66ab2a3e42d9 
Everett, J. A. C., Colombatto, C., Chituc, V., Brady, W. J., \& Crockett, M. (2020). The effectiveness of moral messages on public health behavioral intentions during the COVID-19 pandemic [Preprint]. PsyArXiv. https://doi.org/10.31234/osf.io/9yqs8

Faul, F., Erdfelder, E., Lang, A., \& Buchner, A. (2007). G*Power 3: A flexible statistical power analysis program for the social, behavioral, and biomedical sciences. Behavior Research Methods, 39(2), 175-191. https://doi.org/10.3758/BF03193146

Galea, S., Merchant, R. M., \& Lurie, N. (2020). The mental health consequences of COVID-19 and physical distancing: The need for prevention and early intervention. JAMA Internal Medicine. https://doi.org/10.1001/jamainternmed.2020.1562

Garfin, D. R., Silver, R. C., \& Holman, E. A. (2020). The novel coronavirus (COVID-2019) outbreak: Amplification of public health consequences by media exposure. Health Psychology. Advance online publication. http://dx.doi.org/doi:10.1037/hea0000875

Gray, J. A., \& McNaughton, N. (2000). The neuropsychology of anxiety: An enquiry into the functions of the septo-hippocampal system (2nd ed.). Oxford University Press. http://doi.org/10.1093/acprof:oso/9780198522713.001.0001

Greenberg, J., Solomon, S., \& Pyszczynski, T. (1997). Terror management theory of self-esteem and cultural worldviews: Empirical assessments and conceptual refinements. Advances in Experimental Social Psychology, 29, 61-139. http://www.doi.org/10.1016/S0065$\underline{2601(08) 60016-7}$

Harrop, F. (2020, March 24). The togetherness of social distancing. RealClear Politics. https://www.realclearpolitics.com/articles/2020/03/24/the_togetherness_of_social_distan cing_142749.html

Hayes, A. F. (2018). Introduction to mediation, moderation, and conditional process analysis: A 
regression-based approach (2 $2^{\text {nd }}$ ed.). New York: Guilford Press.

Hayes, J., \& Hubley, C. (2017). Between a rock and a hard place: When affirming life reduces depression, but increases anxiety. Journal of Social and Clinical Psychology, 36(10), 860-882. http://www.doi.org/101521jscp20173610860

Hayes, J., Prentice, M., \& McGregor, I. (2017). Giving in and giving up: Accommodation and fatalistic withdrawal as alternatives to primary control restoration. In M. Bukowski, I. Fritsche, A. Guinote, \& M. Kofta (Eds.), Coping with lack of control in a social world. Routledge.

Hayes, J., Ward, C. L. P., \& McGregor, I. (2016). Why bother? Death, failure, and fatalistic withdrawal from life. Journal of Personality and Social Psychology, 110(1), 96-115. http://www.doi.org/10.1037/pspp0000039

Hilton, S. (2020, March 20). Steve Hilton: Flatten the coronavirus curve, but not the economy, before it's too late. Fox News. https://www.foxnews.com/opinion/steve-hilton-flattencoronavirus-curve-but-not-the-economy

Jonas, E., McGregor, I., Klackl, J., Agroskin, D., Fritsche, I., Holbrook, C., . . Quirin, M. (2014). Advances in experimental social psychology. In J. M.Olson \& M. P.Zanna (Eds.), Threat and defense: From anxiety to approach (pp. 219-286). Academic Press.

Lunn, P. D., Belton, C. A., Lavin, C., McGowan, F. P., Timmons, S., \& Robertson, D. A. (2020). Using behavioral science to help fight the coronavirus. Journal of Behavioral Public Administration, 3(1). http://www.doi.org/10.30636/jbpa.31.147

McNaughton, N., \& Corr, P. J. (2004). A two-dimensional neuropsychology of defense: Fear/anxiety and defensive distance. Neuroscience \& Biobehavioral Reviews, 28(3), 285305. http://www.doi.org/10.1016/j.neubiorev.2004.03.005 
Mutikani, L. (2020). Coronavirus: Over 20 million Americans have now applied for unemployment benefit. World Economic Forum. https://www.weforum.org/agenda/2020/04/united-states-unemployment-claimantscoronavirus-covid19/

Pyszczynski, T., Greenberg, J., Solomon, S., Arndt, J., \& Schimel, J. (2004). Why do people need self-esteem? A theoretical and empirical review. Psychological Bulletin, 130(3), 435-468. http://www.doi.org/10.1037/0033-2909.130.3.435

Rosenberg, M. (1965). Society and the adolescent self-image. Princeton University Press.

Seligman, M. E. P. (1975). Helplessness: On depression, development, and death. Freeman.

Slaughter, G. (2020, March 6). Expert: COVID-19 isn't containable and Canada should focus on protecting seniors. CTV News. https://www.ctvnews.ca/health/expert-covid-19-isn-tcontainable-and-canada-should-focus-on-protecting-seniors-1.4842922

Taub, A. (2020, April 6). A new Covid-19 crisis: Domestic abuse rises worldwide. The New York Times. https://www.nytimes.com/2020/04/06/world/coronavirus-domestic-violence.html

Trump, D. J. [@realDonaldTrump]. WE CANNOT LET THE CURE BE WORSE THAN THE PROBLEM ITSELF. (2020, March 23). [Tweet]. Twitter. https://twitter.com/realDonaldTrump/status/1241935285916782593

United States Department of Labor. (2020). COVID-19 impact. https://www.dol.gov/ui/data.pdf WhiteHouse.gov (2020). 15 days to slow the spread. https://www.whitehouse.gov/articles/15days-slow-spread/

Worldometer. (2020). Covid-19 coronavirus pandemic [Infograph]. Worldometer.com. http://www.worldometers.info/coronavirus/ 
World Health Organization. (2020). WHO Director-General's opening remarks at the media briefing on COVID-19 - 11 March 2020. https://www.who.int/dg/speeches/detail/whodirector-general-s-opening-remarks-at-the-media-briefing-on-covid-19---11-march-2020

Zimbardo, P. G., \& Boyd, J. N. (1999). Putting time in perspective: A valid, reliable individualdifferences metric. Journal of Personality and Social Psychology, 77(6), 1271-1288. http://www.doi.org/10.1037/0022-3514.77.6.1271 


\section{Table 1}

Descriptive Statistics for Scale Items Assessing Fatalism Toward Fighting Covid-19.

Item-total

\begin{tabular}{lcccc} 
Item & Mean & SD & Skewness & Correlation \\
\hline 1. Since whatever will be will be, it doesn't & 2.46 & 1.70 & 1.07 & .73
\end{tabular}
really matter what I do to try to stop covid-19.

2. My actions can contribute to stopping the spread of covid-19.*

3. Staying home can make all the difference in the fight against covid-19.*

4. I often feel that there is no point in even trying to stop the spread of covid-19.

5. I can help to stop the spread of covid-19.*

5.59

5.69

2.48

5.52

5.37

6. I believe that helping to stop covid-19 is within my control.*

7. I have the ability to make decisions that will reduce the spread of covid-19.*

8. What I do now to fight covid-19 matters in the long run.*

9. When thinking about tackling covid-19, I often think "why bother?".

10. It doesn't make sense to worry about covid-19 because there is nothing that I can do about it anyway.

11. It is within my power to help reduce the spread of covid-19.*

12. My actions will make a difference in reducing the death-toll from covid-19.*

13. Social distancing is NOT a good way to fight covid-19.

14. There is no effective way to stop covid-19 from spreading.

15. Forcing people who are not sick into selfisolation will reduce the spread of covid19.*

16. The spread of covid-19 is controlled by forces that I cannot influence.

5.68

5.63

2.41

2.78

5.47

5.41

2.22

3.09

5.48

Scale Average

1.50

1.37

1.32

1.37

$-1.23$

$-0.97$

$1.29 \quad-1.37$

$-.71$

$1.36-1.21$

$-.69$

1.71

1.17

.68

1.74

0.85

.67

$-.71$

71 


\section{Table 2}

Bivariate Correlations Among Fatalism and Negative Emotions

\begin{tabular}{|c|c|c|c|c|}
\hline Variable & 1 & 2 & 3 & 4 \\
\hline Anxiety (1) & --- & & & \\
\hline Fear (2) & $.80^{* * *}$ & --- & & \\
\hline Depression (3) & $.79^{* * *}$ & $.81^{* * *}$ & --- & \\
\hline Insecurity (4) & $.26^{* * *}$ & $.27^{* * *}$ & $.33^{* * *}$ & --- \\
\hline Fatalism & .07 & .02 & $.09^{* *}$ & -.05 \\
\hline Fatalism (partial correlations) & .04 & $-.10^{* *}$ & $.12^{* * *}$ & $-.08^{*}$ \\
\hline
\end{tabular}




\section{Table 3}

Indirect Effects of Messaging Condition on Negative Emotions Through COVID-19 Fatalism.

\begin{tabular}{cccccc}
\hline Message Condition & Outcome & Effect & Boot SE & Boot LLCI & Boot ULCI \\
\hline Fatalistic & Anxiety & .004 & .006 & -.004 & .022 \\
(vs. control) & Fear & -.015 & .009 & -.040 & -.002 \\
& Depression & .018 & .010 & .002 & .044 \\
& Insecurity & -.018 & .013 & -.056 & -.001 \\
\hline Optimistic & Anxiety & -.006 & .007 & -.024 & .006 \\
(vs. control) & Fear & .019 & .010 & .004 & .045 \\
& Depression & -.023 & .012 & -.054 & -.006 \\
& Insecurity & .024 & .015 & .003 & .063 \\
\hline
\end{tabular}

Note. All indirect effects represent unstandardized regression coefficients. Boot $=$ Bootstrapped, $\mathrm{SE}=$ Standard Error, LLCI = Lower Level Confidence Interval, ULCI = Upper Level

Confidence Interval. Confidence intervals represent $95 \% \mathrm{CIs}$, thus intervals that do not contains zero are significant at the $p<.05$ level. 


\section{Figure 1}

Effect of Messaging Condition on Fatalism toward COVID-19

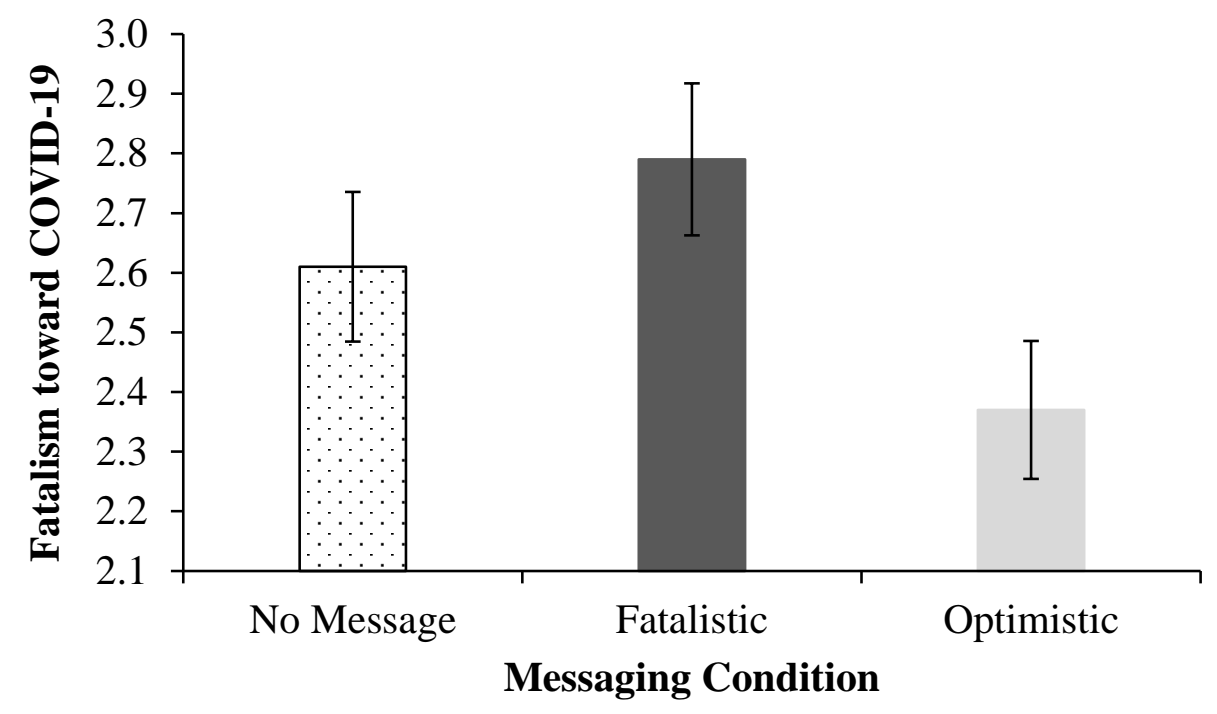

Note. Error bars represent $95 \%$ confidence intervals. 


\section{Figure 2}

Effect of Messaging Condition on Support for COVID-19 Mitigation Efforts

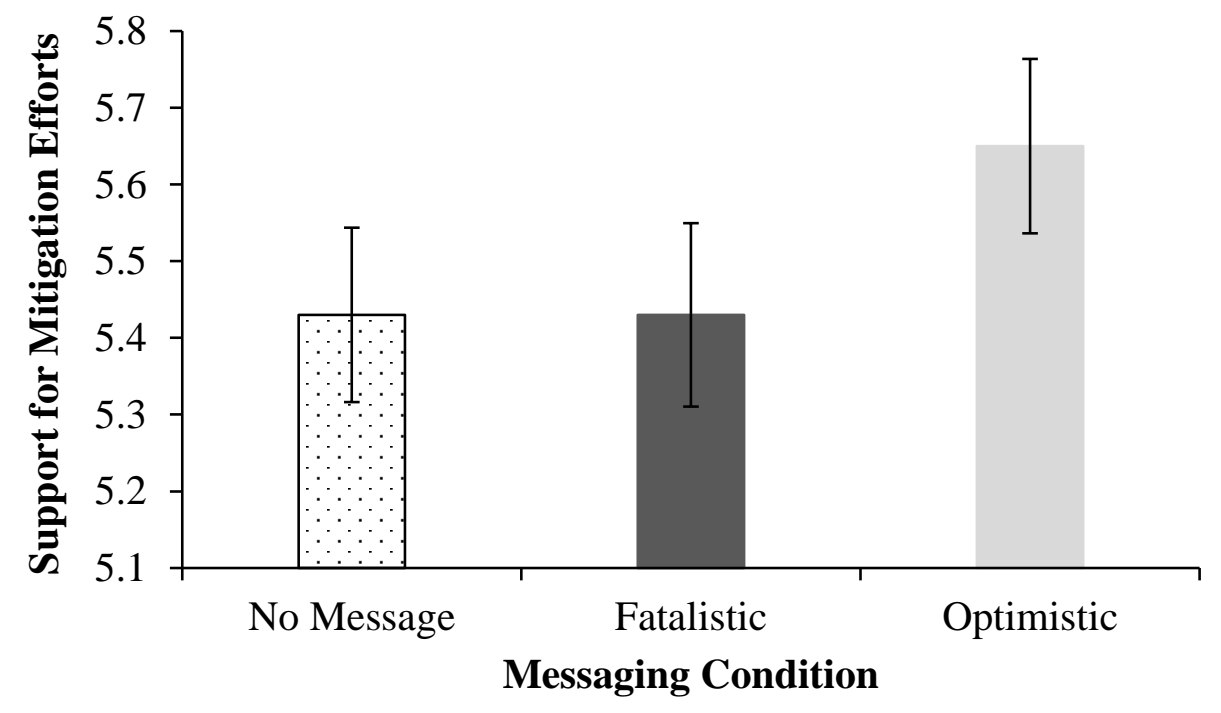

Note. Error bars represent $95 \%$ confidence intervals. 


\section{Figure 3}

Path Model of Indirect and Direct Effects of Messaging Condition on Support for Mitigation Efforts Through the Mediator of Fatalism toward COVID-19

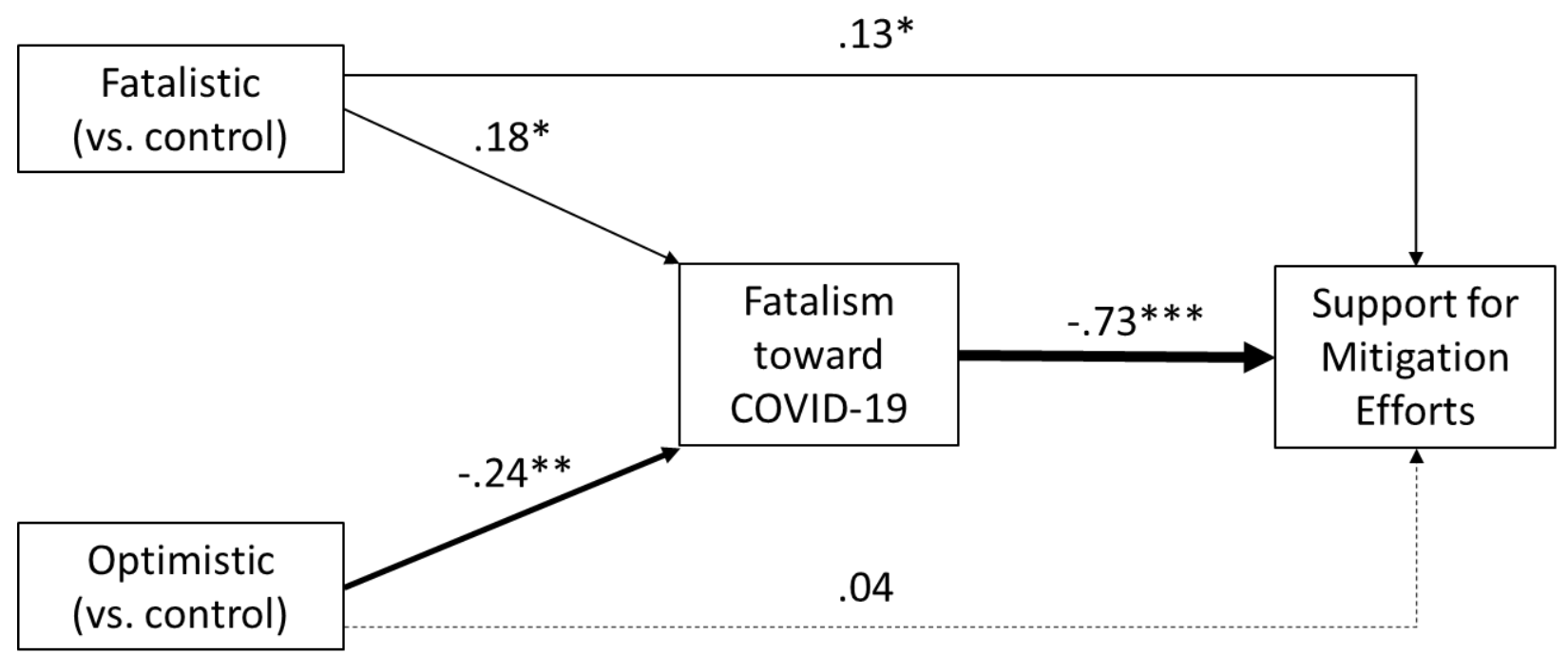

Note. Path coefficients represent unstandardized regression slopes. Broken lines represent nonsignificant effects. Heavier lines represent stronger relationships. ${ }^{*} p<.05^{* *} p<.01^{* * *} p<.001$ 


\section{Figure 4}

Effect of Messaging Condition on Emotional Distress

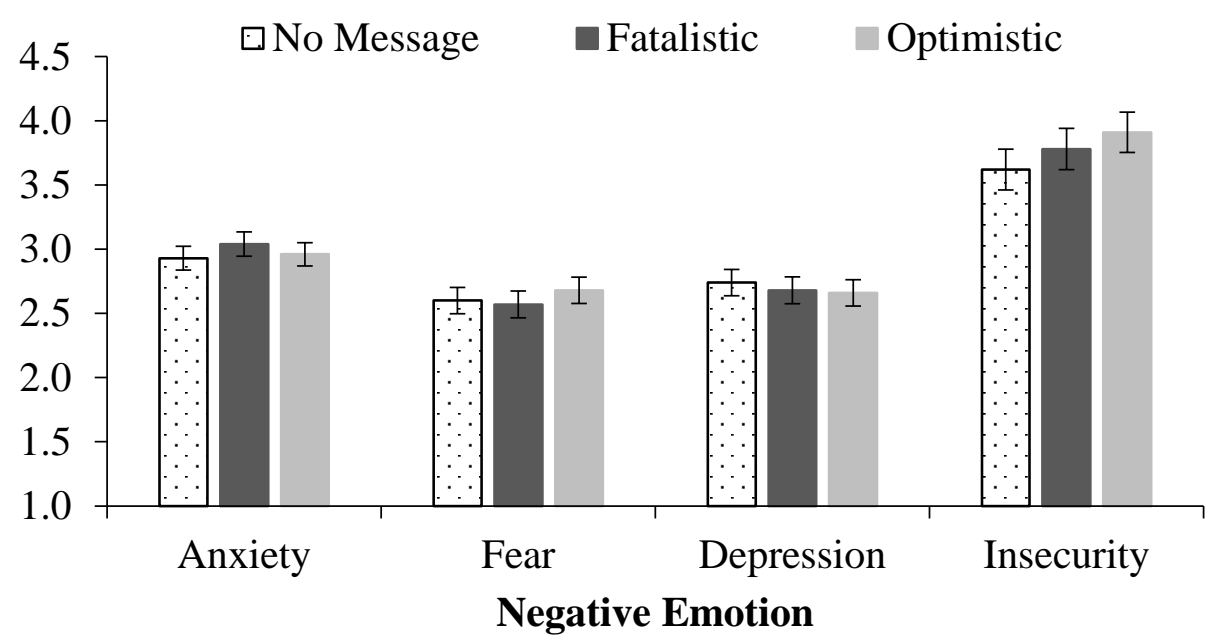

Note. Error bars represent $95 \%$ confidence intervals. Condition means and confidence intervals for each negative emotion represent adjusted values controlling for the remaining three emotions. 


\section{Figure 5}

Path Model of Indirect and Direct Effects of Messaging Condition on Negative Emotions Through the Mediator of Fatalism toward COVID-19

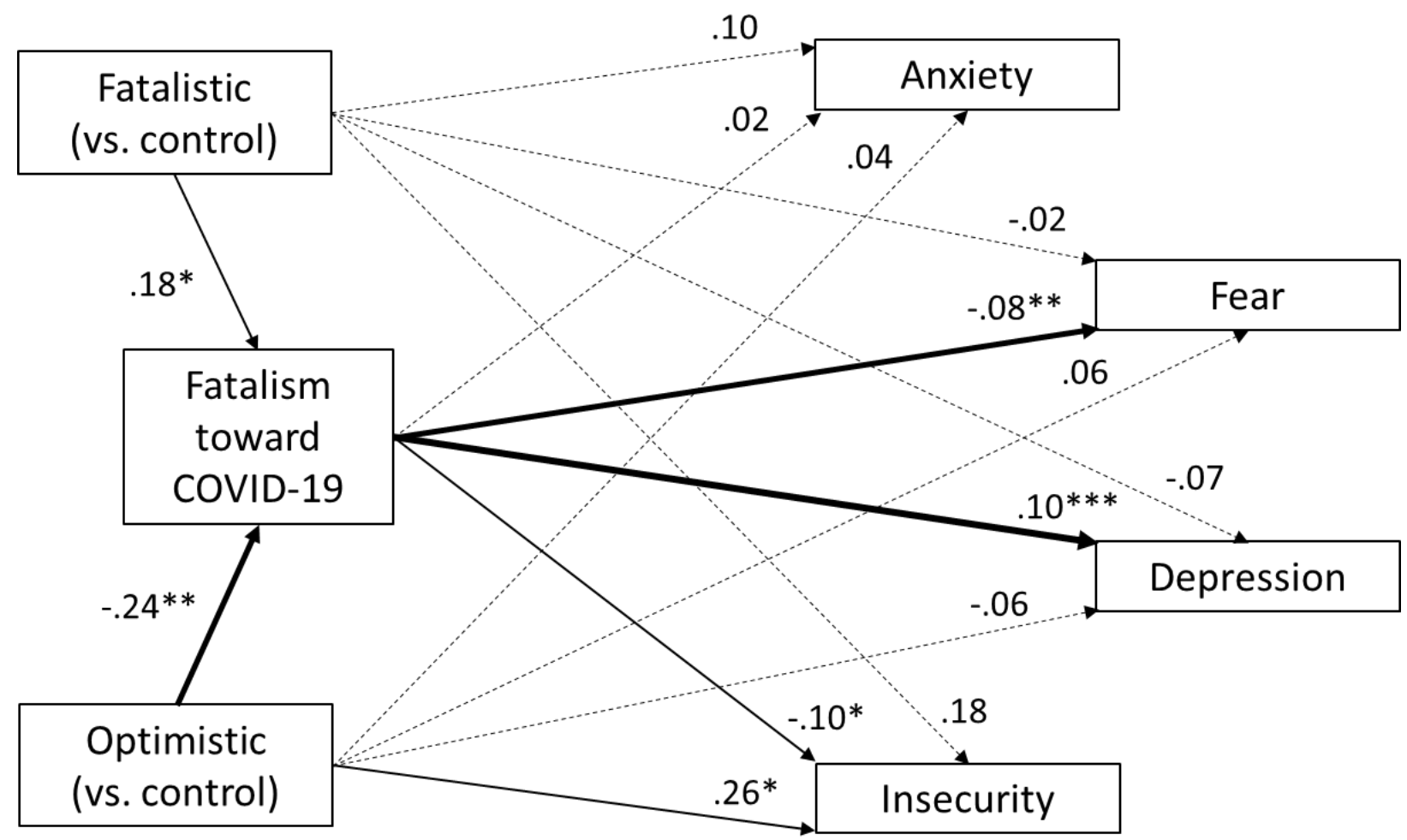

Note. Path coefficients represent unstandardized regression slopes. Broken lines represent nonsignificant effects. Heavier lines represent stronger relationships. ${ }^{*} p<.05{ }^{* *} p<.01{ }^{* * * *} p<.001$ 